Predicting Ideological Prejudice: A Pre-Registered Replication of Brandt (2017)

Mark J. Brandt

Tilburg University

m.j.brandt@tilburguniversity.edu

Draft Date: 25 March 2018

Data \& Code: https://osf.io/g28yc/ (It will be necessary to download the existing data used in this study from other sources. These sources are noted in a "read me" file on the OSF page.)

This is a preprint. Maybe it'll be published one day. Feedback is appreciated. 


\begin{abstract}
Brandt (2017) demonstrated that a simple model using the perceived ideology of a target group can predict the size and direction of the association between political ideology and prejudice. A meta-analysis of four studies showed that this simple ideology only model performs better than a null model and models using the perceived conventionalism, status, and choice of being a member of the target groups. This report describes a preregistered replication which uses data from the American National Election Study's 2016 Time Series ( $N=2616)$, archival data that was only made available after the study was preregistered. The findings of Brandt (2017) were replicated and the meta-analysis reported in Brandt (2017) is updated with these new data.
\end{abstract}




\section{Predicting Ideological Prejudice: A Pre-Registered Replication of Brandt (2017)}

Brandt (2017) tested how several characteristics of target groups can be used to predict the size and direction of the association between ideology and prejudice. The model that worked the best across four studies (including a meta-analysis) was a model that only included the perceived ideology of the target group. By combining the perceived ideology of a target group with a simple linear model, it was possible to anticipate the size and direction of the ideology-prejudice association in new data. This ideology only model was more accurate than models that used the perceived status of the target groups, the perceived choice of being a member of the target groups, or the perceived conventionalism of the target group (The latter comparison was only significant in the meta-analysis and not in any individual study). This is important because it shows that accurate predictions can be made using relatively simple models and that theories that expect that status (Sidanius \& Pratto, 2001) or choice (Hodson \& Dhont, 2015) account for the ideology-prejudice association do not make as accurate of predictions as those that focus on ideological characteristics (Brandt et al., 2014). It also provides a clear benchmark for other alternative models to be tested against.

The purpose of this study is to replicate the model testing phase of Brandt (2017) with new data. I use the models developed in the model building phase of Brandt (2017) and compare those predictions with the observed ideology-prejudice associations in the 2016 Time Series of the American National Election Study (2017). This allows us to know if the models built in Brandt (2017) are predictive in an new political and election context that included increasing levels of affective polarization (Pew, 2016; cf. lyengar \& Westwood, 2015) and saw the rise of new political movements (e.g., the Alt Right; Forscher \& Kteily, 2017; Schreckinger, 2017). It is a clear opportunity to assess the predictive validity of a model aimed at making accurate predictions.

\section{Model Testing}

I used all of the available data from the 2016 Time Series of the American National Election Study (31 March 2017 version). This is a representative sample of Americans collected around the time the 2016 national elections. It included the necessary data from 2616 participants (1260 men, 1356 women), which allows us to make accurate parameter estimates (e.g., $\sim 98$ power to detect $r=.08$ ). Another advantage of this dataset is that the codebook was available prior to the availability of the data to the scholarly community. This allowed me to pre-register my analysis plan without first seeing the dataset or any other results based on the same data (https://aspredicted.org/blind.php/?x=9my46c).

The study used all of the same measures as Brandt (2017). ${ }^{1}$ Expressed prejudice was measured with feeling thermometers that ranged from 0 (cold/ unfavorable) to 100 (warm/favorable) towards 21 different social groups (a $22^{\text {nd }}$ group, Black Lives Matter protestors was not included because I did not have an estimate of its perceived group characteristics that are needed to make a prediction); ratings were reverse-scored so that

\footnotetext{
${ }^{1}$ Some of the covariates were measured on slightly different scales in this dataset compared to the dataset used in the original paper.
} 
higher scores indicated more prejudice. Participants' self-reported ideology was measured with a single item on a 1 to 7 scale, where higher scores indicated more conservatism. Age (split across 13 categories), gender (women $=-.5$, men $=.5$ ), years of education (less than 1 $=1^{\text {st }}$ grade to $16=$ doctorate degree), race (Contrast $1:-0.75=$ White, $0.25=$ Black, Hispanic, and other; Contrast 2: $0=$ White, $0.33=$ Black and Hispanic, $-0.66=$ other; Contrast 3: $0=$ White and other, $0.5=$ Black, $-0.5=$ Hispanic $)$, and income $(1=\$ 100,000-109,999$ to $6=$ $\$ 250,000$ or more) were included as covariates. As in the original study, all variables were recoded to range from 0 to 1 . Age, education, and income were mean-centered. Gender and race-ethnicity contrast codes were also mean-centered to weight these contrasts at their average (Hayes, 2013).

To estimate the observed ideology-prejudice association, I regressed prejudice on participants' ideology and covariates (age, gender, education, race, income) for each of the different target groups. Then I compared the observed ideology estimates with the predicted estimates from the models tested in Brandt (2017). Both the observed estimates and the predicted estimates are in Figure 1.

\section{Results}

The pre-registration specified that the key analysis is a one-way ANOVA comparing the ideology-only, status-only, choice-only, and null models. This ANOVA was significant, $F(3$, $80)=3.20, M S E=.03, p=.03$, and the ideology-only model was significantly more accurate than the other three models ( $p^{\prime}$ s $\left.[.009, .02]\right)$. The pre-registered secondary analysis was an ANOVA that compared all of the models used in in Brandt (2017). This ANOVA was also significant, $F(6,140)=3.59, M S E=.02, p=.002$. As can be seen in Figure 2 , the ideology only model was a significant improvement over the status-only, choice-only, and null models. As specified in the pre-registration, the data from this new study was added the meta-analysis reported in Brandt (2017). It shows that the ideology only model is the most accurate model of all of the models tested (see Figure 3).

\section{Discussion}

These results fully replicate the findings of Brandt (2017). A meta-analysis of all studies assessing the accuracy of these models finds that both the ideology only model and the ideology, status, and choice model are the most accurate models. Because these two models are equally accurate and the ideology only model is the most parsimonious, the ideology only model is considered the best model. Models involving status and choice do not make accurate predictions. For example, in the meta-analysis the ideology only model is significantly more accurate than the status only model (difference $=-0.06, S E=0.01, z=-$ $5.39, p<.001)$. This difference in mean squared error is the difference between finding a slope of $b=0$ and a slope of $b=.24$ (or a slope of $b=.24$ and a slope of $b=.48$, etc.). These are substantially different predictions. If you need to anticipate the size and direction of the association between ideology and prejudice, you need to know the perceived ideology of the target group.

The current replication study does not solve any of the limitations of the original study. However, now that we know that the model replicates well in a sample from the United 
States, a natural next step is to see how well the model replicates in samples outside of the United States. Similarly, now that we know the model replicates well using feeling thermometers to assess prejudice, a natural next step is to see how well the model replicates when using alternative measures of prejudice (e.g., social distance preferences, automatic assessments). This replication study provides a solid foundation for the continued test of the ideology only model to predict the association between ideology and prejudice.

\section{References}

American National Election Studies. (2017). The ANES 2016 Pilot Study [Data file]. Stanford University and the University of Michigan (Producers). Retrieved from http://www.electionstudies.org/studypages/anes_timeseries_2016/anes_timeseries_ 2016.htm

Brandt, M. J. (2017). Predicting ideological prejudice. Psychological Science, 28, 712-722.

Brandt, M. J., Reyna, C., Chambers, J. R., Crawford, J. T., \& Wetherell, G. (2014). The ideological-conflict hypothesis: Intolerance among both liberals and conservatives. Current Directions in Psychological Science, 23, 27-34.

Forscher, P. S., \& Kteily, N. (2018, January 23). A Psychological Profile of the Alt-Right. Retrieved from psyarxiv.com/c9uvw

Hayes, A. F. (2013). Introduction to mediation, moderation, and conditional process analysis: A regression-based approach. New York, NY: Guilford Press

Hodson, G., \& Dhont, K. (2015). The person-based nature of prejudice: Individual difference predictors of intergroup negativity. European Review of Social Psychology, 26, 1-42.

lyengar, S., \& Westwood, S. J. (2015). Fear and loathing across party lines: New evidence on group polarization. American Journal of Political Science, 59, 690-707.

Pew Research Center (2016, June). Partisanship and political animosity in 2016: Highly negative views of the opposing party - and its members. Retrieved from http://www.people-press.org/2016/06/22/partisanship-and-political-animosity-in2016/

Schreckinger, B. (2017, January/February). The Alt-Right Comes to Washington. Politico Magazine. Retrieved from http://www.politico.com/magazine/story/2017/01/altrighttrump-washington-dc-power-milo-214629

Sidanius, J., \& Pratto, F. (2001). Social dominance: An intergroup theory of social hierarchy and oppression. Cambridge, England: Cambridge University Press. 


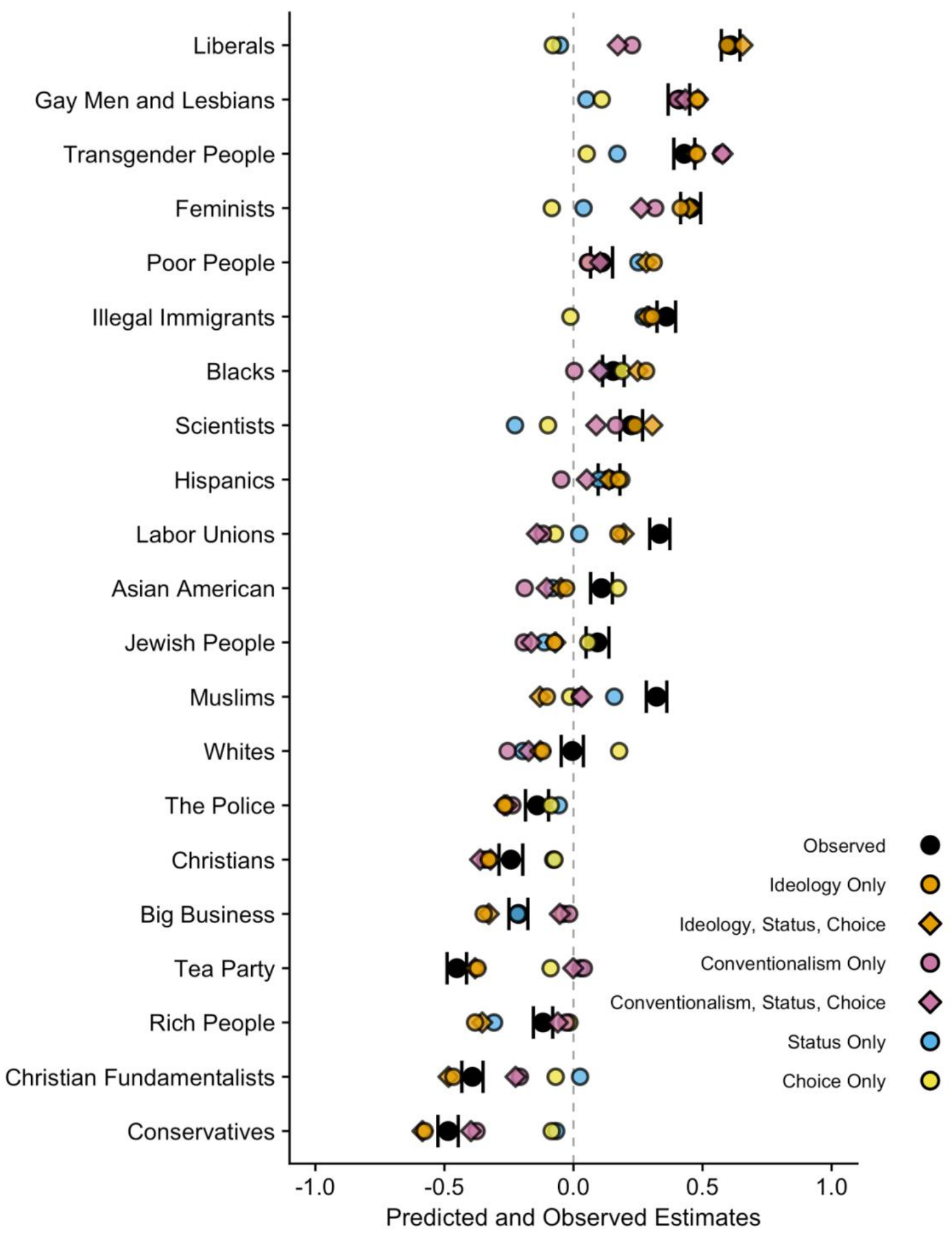

Figure 1. Comparison of the predicted and observed estimates for the association between ideology and prejudice toward the target groups. The observed estimates were obtained using ordinary least squares regression, adjusting for age, gender, education, income, and race-ethnicity. The predicted estimates were obtained from the models described in Brandt (2017). The dashed vertical line represents the predictions of the null model. All variables were coded to have a range of 1. Error bars represent 95\% confidence intervals. The target groups are ordered from the group perceived as most liberal (top) to the group perceived as most conservative (bottom). 


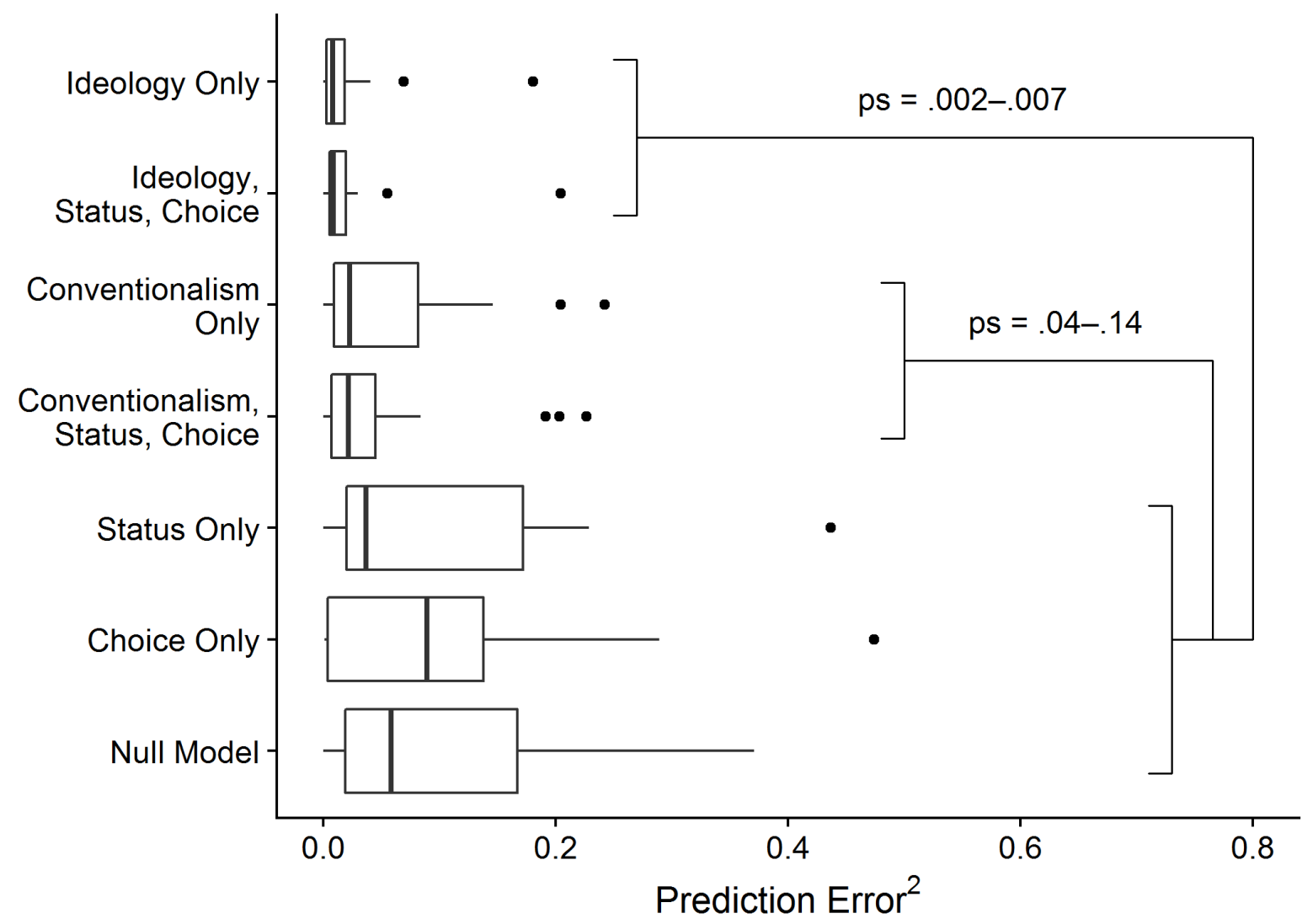

Figure 2. Box plots of the seven models' squared prediction error. The right and left edges of the box indicate the 75th and 25th percentiles, respectively, and the black line near the middle of the box is the 50th percentile. The whiskers represent the lowest and highest data points within 1.5 times the interquartile range of the lowest quartile and the highest quartile, respectively. The circles represent outliers. The ranges of $p$ values indicate the values obtained when the two ideology models and the two conventionalism models were compared individually with the status-only, choice-only, and null models. 


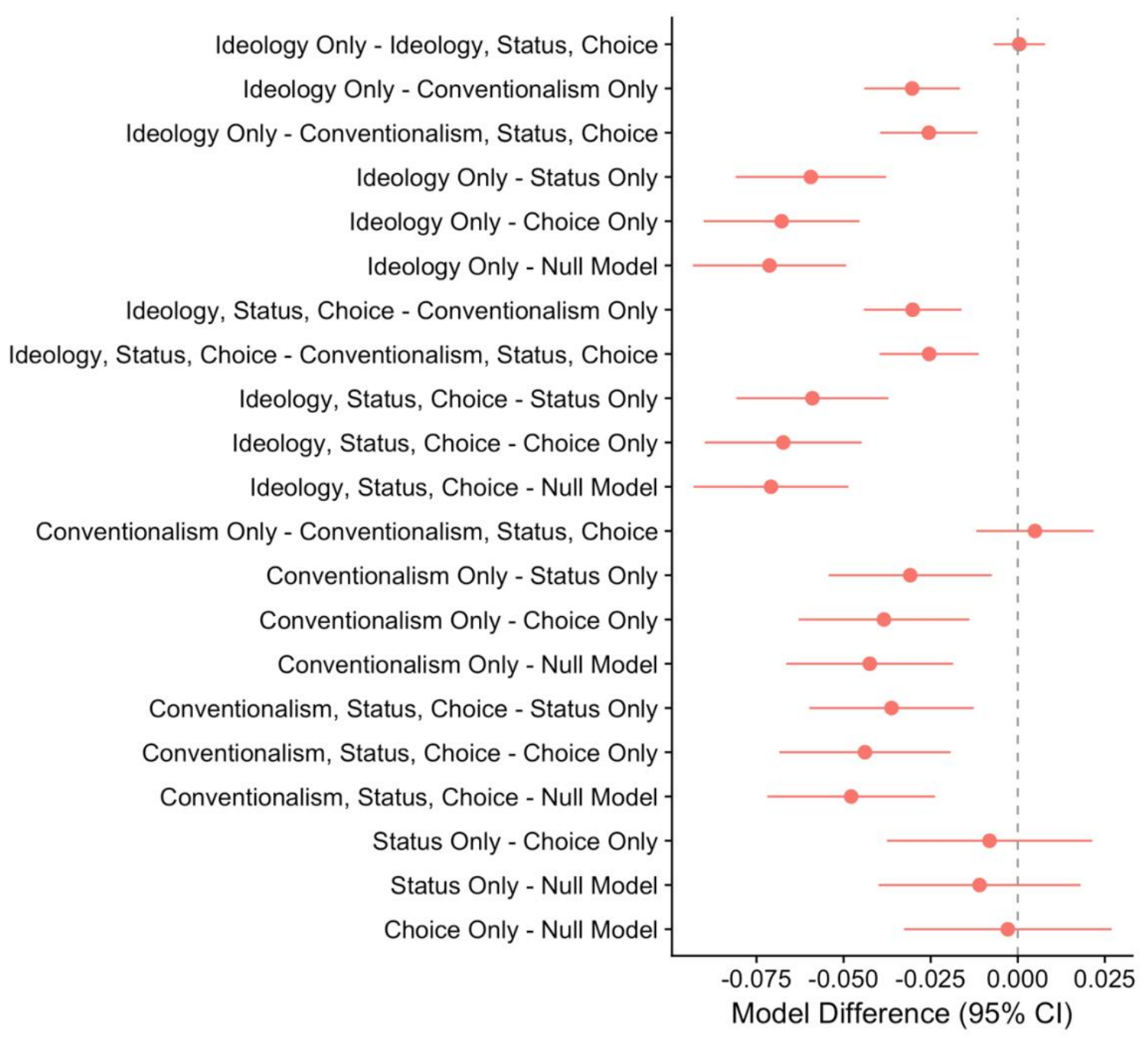

Figure 3. Meta-analytic comparison of models' MSE. Data is from the current study and Brandt (2017). X-axis is the estimated difference between the two models and the error bars are $95 \%$ confidence intervals. The vertical dashed line indicates no difference between the models. 